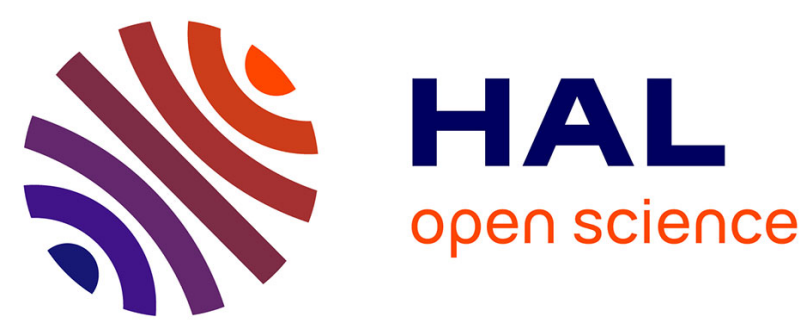

\title{
Rééducation pelvi-périnéale et troubles de la statique pelvienne de la femme
}

Thibault Thubert, Els Bakker, Xavier Fritel

\section{To cite this version:}

Thibault Thubert, Els Bakker, Xavier Fritel. Rééducation pelvi-périnéale et troubles de la statique pelvienne de la femme. Gynécologie Obstétrique \& Fertilité, 2015, 43 (5), pp.non connue à ce jour. 10.1016/j.gyobfe.2015.03.026 . inserm-01150249

\section{HAL Id: inserm-01150249 https://www.hal.inserm.fr/inserm-01150249}

Submitted on 9 May 2015

HAL is a multi-disciplinary open access archive for the deposit and dissemination of scientific research documents, whether they are published or not. The documents may come from teaching and research institutions in France or abroad, or from public or private research centers.
L'archive ouverte pluridisciplinaire HAL, est destinée au dépôt et à la diffusion de documents scientifiques de niveau recherche, publiés ou non, émanant des établissements d'enseignement et de recherche français ou étrangers, des laboratoires publics ou privés. 
1 Rééducation pelvipérinéale et troubles de la statique pelvienne de la femme

2 Pelvic floor muscle training and pelvic floor disorders in women

3 Point de vue d'expert

4

5 Thibault THUBERT, 1

6 Els BAKKER, 2

7 Xavier FRITEL, 3

8

9 1, Service de Gynécologie-Obstétrique et Médecine de la Reproduction, Hôpital Antoine

10 Béclère 157 rue de la porte de Trivaux, F-92140 Clamart, France

11 2, Unité Recherche, HE L de Vinci ; Parnasse-ISEI. 84, Avenue Mounier, B-1200 Bruxelles,

12 Belgique

13 3, Service de Gynécologie-Obstétrique et Médecine de la Reproduction, CHU de Poitiers,

142 rue de la Milétrie, F-86000 Poitiers, France

15

16 Auteur correspondant

$17 \quad$ Pr Xavier Fritel

18 Service de Gynécologie-Obstétrique et Médecine de la Reproduction, CHU de Poitiers, 2 rue

19 de la Milétrie, 86000 Poitiers, France

20 Tel : 0549443945

21 Fax : 0549443910

22 Mail : xavier.fritel@univ-poitiers.fr

23

24

25 
Résumé

27 Notre objectif est de faire le point sur les résultats de la rééducation périnéale dans le

28 traitement de l'incontinence urinaire et des symptômes du prolapsus génital.

29 Le renforcement des muscles du plancher pelvien permet de réduire les symptômes de

30 l'incontinence urinaire. La rééducation encadrée par un rééducateur permet une

31 guérison dans plus de la moitié des cas de l'incontinence urinaire d'effort. La

32 visualisation de la contraction grâce au biofeedback améliore les résultats, mais cet effet

33 pourrait être expliqué par une prise en charge plus intense et prolongée avec le

34 rééducateur. La place de l'électrostimulation reste à préciser. Les résultats obtenus

35 grâce à l'utilisation des cônes vaginaux sont équivalent au renforcement musculaire avec

36 ou sans biofeedback ou à l'électrostimulation. On ne sait pas si l'effet de la rééducation

37 périnéale avec renforcement musculaire a encore un effet après un an. Dans

38 l'incontinence urinaire d'effort, la rééducation périnéale encadrée par un rééducateur en

39 première intention évite l'intervention chirurgicale dans la moitié des cas à un an.

40 La rééducation périnéale est le traitement de première intention de l'incontinence

41 urinaire du postpartum. Son effet préventif est incertain.

42 Le renforcement des muscles du plancher pelvien semble réduire les symptômes 43 associés au prolapsus génital.

44 En conclusion, la rééducation périnéale encadrée par un rééducateur est un traitement 45 efficace, à court terme, pour réduire les symptômes d'incontinence urinaire ou de 46 prolapsus génital. 
Abstract

49 Our goal is to provide an update on the results of pelvic floor rehabilitation in the

50 treatment of urinary incontinence and genital prolapse symptoms.

51 Pelvic floor muscle training allows a reduction of urinary incontinence symptoms. Pelvic

52 floor muscle contractions supervised by a healthcare professional allow cure in half

53 cases of stress urinary incontinence. Viewing this contraction through biofeedback

54 improves outcomes, but this effect could also be due by a more intensive and prolonged

55 program with the physiotherapist. The place of electrostimulation remains unclear. The

56 results obtained with vaginal cones are similar to pelvic floor muscle training with or

57 without biofeedback or electrostimulation. It is not known whether pelvic floor muscle

58 training has an effect after one year. In case of stress urinary incontinence, supervised

59 pelvic floor muscle training avoid surgery in half cases at 1 year follow-up.

60 Pelvic floor muscle training is the first-line treatment of postpartum urinary

61 incontinence. Its preventive effect is uncertain.

62 Pelvic floor muscle training may reduce the symptoms associated with genital prolapse.

63 In conclusion, pelvic floor rehabilitation supervised by a physiotherapist is an effective

64 short term treatment to reduce the symptoms of urinary incontinence or pelvic organ

65 prolapse.

66 
67

68 recommandations du CNGOF [3].

De nombreuses femmes présentent des troubles urogynécologiques parmi lesquels figurent l'incontinence urinaire à l'effort (IUE), l'incontinence urinaire par urgenturie (IUU), l'incontinence urinaire mixte (IUM), le syndrome d'hyperactivité vésicale, ou bien encore les symptômes d'un prolapsus génital [1]. Selon les dernières recommandations de l'AFU (Association Française d'Urologie) et du CNGOF (Collège National des Gynécologues et Obstétriciens Français), le traitement de première intention de ces troubles repose sur la rééducation pelvi-périnéale [2,3]. La rééducation pelvi-périnéale encadrée par un rééducateur est multimodale comportant différentes facettes : une partie cognitive (éducation, prise de conscience du plancher pelvien), une partie comportementale d'entrainement vésical (modification des habitudes mictionnelles), une partie de renforcement musculaire (contractions volontaires contre résistance avec ou sans biofeedback et électrostimulation), et aussi un travail sur la posture (équilibre et position du bassin). La rééducation des muscles du plancher pelvien a pour but d'augmenter la force, l'endurance, la rapidité de contraction et ensuite d'améliorer la synchronisation. La contraction isolée permet une correction des symptômes, en particulier en cas d'urgenturie, grâce au réflexe périnéo-détrusorien inhibant (la contraction du plancher pelvien inhibe la contraction vésicale), alors que l'automatisation de la contraction avant l'effort va permettre une amélioration des symptômes en cas d'incontinence à l'effort. Notre objectif est de faire le point sur les résultats attendus de la rééducation pelvi-périnéale depuis les dernières 
Dans une récente revue de la littérature, Deffieux et al. rappelaient les différents mécanismes physiopathologiques d'action de la rééducation s'avérant efficace sur l'incontinence urinaire de la femme [4]. Ils analysaient séparément les exercices de contractions périnéales, le biofeedback, l'électrostimulation, et la thérapie comportementale. Il en ressortait que les exercices de contraction volontaire des muscles pelvi-périnéaux (MPP, pelvic floor muscles en anglais) sont associés à une augmentation significative de la force de contraction de ces muscles et à une amélioration des scores d'incontinence urinaire pour les femmes présentant une IUE. En revanche, cette augmentation de la force des MPP n'est pas associée à une augmentation de la pression de clôture urétrale maximum (PCUM), ou à une correction de l'hypermobilité urétrale [4]. L'utilisation de l'électrostimulation vaginale s'accompagne, suivant les modalités des paramètres choisis, d'une augmentation de la force de contraction des muscles pelvi-périnéaux, ou d'une diminution de l'intensité des contractions détrusoriennes, sans modification de la PCUM. Les données disponibles dans la littérature sont plus limitées sur le biofeedback et les thérapies comportementales. Une étude visant à évaluer les modifications morphologiques des muscles des releveurs de l'anus avant et après un programme de séances de contractions périnéale chez des patientes présentant une IUE, montrait une réduction du hiatus des releveurs [5].

En 2014, Dumoulin et al. ont actualisé les données de la dernière méta-analyse publiée en 2010 visant à comparer l'efficacité d'un programme de contractions volontaires des MPP encadré par un rééducateur par rapport à un groupe contrôle chez les femmes souffrant d'IUE [6,7]. Après exclusion des études de faible pertinence, 18 essais contrôlés randomisés (1051 patientes) ont été inclus dans cette nouvelle méta- 
analyse. Les auteurs concluent que les femmes présentant une IUE isolée et bénéficiant

116 d'une rééducation périnéale de renforcement musculaire encadrée par un rééducateur

117 rapportaient un taux de guérison subjective de leur IUE 8 fois supérieur comparé au

118 groupe contrôle (RR 8,38 [IC95\% 3,68-19,07], 46/82 (56,1\%) versus 5/83 (6,0\%)), un

119 taux de guérison objective 7 fois supérieur (RR 7,5 [2,89-19,47], 38/71 (53,5\%) versus

120 4/64 (6,3\%)), une réduction supérieure de la quantité des fuites mesurée par le pad-test

121 (RR -13.22 [de -26.36 à -0.09]), et un taux d'amélioration des symptômes ou de guérison

12217 fois supérieur au groupe contrôle (RR 17,33 [4,31-69,64], 32/58 (55\%) versus 2/63

$123(3.2 \%))$. Il n'était pas possible de tirer des conclusions pour les patientes présentant une

124 IUU isolée ou une IUM. En considérant tous les types d'IU confondus, le taux de guérison

125 était 5,5 fois supérieur dans le groupe rééducation comparé au groupe contrôle (RR 5,55

126 [2,87-10,52]), le taux de guérison ou d'amélioration des symptômes était 2,3 fois

127 supérieur au groupe contrôle (RR 2,35, [1,62-3,39]). L'efficacité à long terme (plus d'un

128 an après son arrêt) de la rééducation pelvi-périnéale n’a pas pu être évaluée

129 convenablement car seules deux études rapportaient ces données. Les principales

130 limites de cette méta-analyse reposaient sur la méthodologie des essais inclus : ils

131 étaient tous de petites ou moyennes tailles avec une description aléatoires des critères

132 d'inclusion et des protocoles de rééducation peu décrits et bien souvent différents [8].

133 La rééducation pelvi-périnéale basée sur le renforcement musculaire encadrée par un

134 rééducateur est donc efficace sur l'incontinence urinaire. En l'absence de données à long

135 terme, il n'est pas certain que cet effet soit durable après un an. A notre avis, il n'est pas

136 possible de préciser quel est le programme de rééducation le plus efficace.

Dans un essai randomisé pourtant sur l'IUE prédominante, Labrie et al. ont

138 comparé la chirurgie par bandelette sous-urétrale et la rééducation [9]. Les patientes

139 étaient autorisées à changer de groupe de traitement au cours du suivi de 12 mois. 
140 L'analyse en intention de traiter montrait que la chirurgie était plus efficace que la 141 rééducation (85\% de guérison subjective versus 53 et $76 \%$ de guérison objective versus 142 58). A un an, 49\% des femmes du groupe rééducation avaient opté pour la chirurgie. Ce 143 qui revient à dire que la rééducation en première intention évite la chirurgie dans la 144 moitié des cas à un an. L'apport de la visualisation de la contraction des MPP grâce au biofeedback a été 146 évalué par une méta-analyse réalisée en 2011 par Herderschee [10]. Parmi les 24 essais 147 randomisés inclus (1583 patientes), seuls 7 (250 vs. 270 patientes) ont étudié le critère 148 de jugement principal, et seuls deux utilisaient les mêmes protocoles. Selon les mesures 149 de risque de biais, les niveaux de preuves étaient faibles à modérés. Les études 150 démontraient un taux d'amélioration et de guérison des symptômes d'IU rapportés par 151 les patientes plus important dans le groupe combinant la visualisation de la contraction 152 et le renforcement des MPP (RR 0,75 [0,66-0,86]). L'association des deux modalités de 153 traitement était également corrélée à une diminution du nombre d'épisodes journaliers 154 de fuite d'urine $(-0,12[-0,22--0,01])$. L'un des biais majeur de cette analyse résidait sur 155 la disparité des programmes de rééducation musculaire périnéale. La différence 156 observée pourrait s'expliquer par une meilleure conscientisation, et donc une 157 normalisation plus rapide de l'inscription corticale des MPP [11]. Il n'est toutefois pas 158 certain que l'amélioration des symptômes soit directement du ressort du biofeedback 159 mais pourrait également s'expliquer par le fait que les patientes consultent plus 160 fréquemment leur rééducateur en cas de biofeedback. C'est-à-dire que les programmes 161 de rééducation avec biofeedback sont plus prolongés que les programmes sans 162 biofeedback alors que l'on sait que les programmes de rééducation de renforcement 163 musculaire prolongés sont plus efficaces que les programmes de durée standard, 
164 probablement parce que la prolongation de l'encadrement par le rééducateur favorise 165 l'adhésion [12].

166 L'utilisation de cônes vaginaux pour le traitement de l'incontinence urinaire a été 167 évaluée par une méta-analyse en 2013 [13]. Cette analyse incluait 23 essais contrôlés 168 randomisés (1806 patientes dont 717 avaient utilisés des cônes vaginaux). Si les auteurs 169 montraient un avantage significatif à l'utilisation des cônes par rapport à l'absence de 170 traitement pour la guérison de l'IU, ils ne montraient pas de différence significative 171 entre l'utilisation de cônes et une rééducation de renforcement musculaire du périnée 172 (RR 1,01 [0,91-1,13]) ainsi qu'entre l'utilisation de cônes et l'électrostimulation (RR 1,26 $173[0,85-1,87])$. Ils ne retrouvaient pas non plus de différence entre la combinaison d'une 174 rééducation musculaire périnéale avec l'utilisation de cônes et une rééducation 175 musculaire périnéale sans cônes. Le niveau de preuve de ces analyses était faible en 176 raison d'une disparité des critères d'inclusion des patientes (incontinence rapportée, 177 incontinence définie à l'urodynamique), des critères de jugement (qualité de vie, 178 guérison urodynamique, guérison rapportée par la patiente...), et de la méthodologie 179 (différents programmes de rééducation selon les études). La revue systématique d'Imamura et al. de 2010 montre que l'électrostimulation 181 (versus absence de traitement) améliore l'incontinence urinaire (OR 3,93 [1,43-10,80]) 182 mais ne s'accompagne pas de plus de guérisons (OR 1,10 [0,41-2,94]). La rééducation 183 périnéale de renforcement musculaire semble plus efficace que l'électrostimulation en 184 terme d'amélioration (OR 2,18 [0,76-6,28]) et de guérison (OR 2,65 [0,82-8,60]) de 185 l'incontinence sans que la différence ne soit significative. La combinaison de 186 l'électrostimulation et d'une rééducation de renforcement musculaire du périnée est 187 plus efficace que l'absence de traitement en terme d'amélioration de l'incontinence (OR 8,69 [1,87-40,32]), en revanche il n'existait pas de différence significative en terme de 
guérison de l'incontinence (OR 1,76 [0,27-11,54]) [12]. La dernière revue de l'ICI

190 (International Consultation on Incontinence) ne retrouve pas de différence entre

191 rééducation périnéale avec et rééducation sans électrostimulation [14]. La place de

192 l'électrostimulation dans la rééducation périnéale reste donc à déterminer. L'absence de

193 critères prédictifs identifiés ne permet pas de déterminer quelles femmes pourraient

194 tirer bénéfice de l'électrostimulation. Il existe des sondes d'électrostimulation vaginale

195 destinées à être utilisées à domicile par les femmes. Malheureusement on manque

196 encore de données probantes sur l'efficacité de ces sondes. Une étude a comparé après

197 rééducation périnéale pour IUE prédominante, un groupe avec l'électrostimulation

198 vaginale à domicile et un groupe sans [15]. Mais, à notre avis, un nombre de perdues de

199 vues à 6 mois 3 fois plus important dans le groupe électrostimulation et des

200 prescriptions additionnelles différentes dans les 2 groupes, empêchent toute conclusion

201 sur l'efficacité de ces sondes.

202 Quelques essais randomisés ont montré que les thérapies comportementales sont

203 plus efficaces que l'absence de traitement dans la prise en charge du syndrome

204 d'hyperactivité vésicale, et de l'IUM chez la femme [16]. A notre avis, ces données

205 restent cependant très limitées et ne permettent pas de conclusion définitive. Deux

206 essais randomisés ne retrouvaient pas de différence entre la rééducation pelvi-périnéale

207 de renforcement musculaire et la thérapie comportementale chez les patientes

208 présentant une IUU ou IUM [16]. Un essai randomisé plus récent portant sur l'IUE chez

209 des patientes âgées de plus de 65 ans, retrouve, en revanche, un avantage significatif à la

210 rééducation pelvi-périnéale de renforcement musculaire (20 semaines de traitement)

211 par rapport à la thérapie comportementale en terme de fuites d'urines à l'effort de toux

212 et de qualité de vie [17]. Les experts de la cinquième conférence de l'ICI recommandent

213 en traitement de première intention, pour les patientes présentant une IUU ou une IUM, 
214 une rééducation pelvi-périnéale de renforcement musculaire ou une thérapie 215 comportementale [16]. Pour les patientes présentant une IUE, la rééducation pelvi216 périnéale de renforcement musculaire est à privilégier en première intention [16]. A 217 notre avis, l'utilisation du renforcement musculaire en cas d'IUE prédominante n'exclue 218 pas d'y associer une part de thérapie comportementale, la place de celle-ci sera fonction 219 du bilan initial fait par le rééducateur et mériterait d'être précisée par des travaux 220 futurs.

La méta-analyse de Wallace et al. a analysé l'efficacité d'une thérapie comportementale par rapport aux anticholinergiques dans la prise en charge de l'IUU.

223 Les auteurs concluaient que les deux traitements avaient un effet thérapeutique proche 224 avec cependant un peu plus de guérison subjective 6 mois après la fin du traitement 225 dans le groupe thérapie comportementale (RR 1,69 [1,21-2,34], 26/27 versus 16/28) et 226 l'absence d'effets secondaires à type de sècheresse buccale dans ce groupe [18]. La 227 thérapie comportementale est donc une alternative thérapeutique en cas d'IUU en 228 particulier chez le sujet âgé qui ne peut pas recevoir d'anticholinergiques.

230 automatique des MPP chez les patientes souffrant d'IUE, et les publications de Griffiths 231 sur la compensation corticale adaptive par rapport à l'IUU ont été à la base de nouvelles 232 approches de rééducation au cours des dernières années [19-21]. Plusieurs 233 rééducateurs proposent une approche globale incorporant à côté du travail musculaire, 234 une correction de la posture, un travail sur d'équilibre, et une prise en charge 235 psychologique et cognitive [22]. Baker et al. ont réalisé un essai contrôlé comparant le 236 yoga et une thérapie anxiolytique (mindfulness therapy) chez des patientes présentant 237 une IUM à composante d'urgenturie prédominante [23]. Ils montraient une plus grande 238 efficacité de la thérapie anxiolytique en nombre de fuites d'urine (pourcentage de fuites) 
239 à 8 semaines, 6 mois et un an. Il s'agissait d'un essai pilote sur un faible effectif avec un 240 taux de perdu de vue important nécessitant confirmation (suivi à un an 12 patiente dans

241 le groupe traitement et 9 patientes dans le groupe yoga). Dans une étude de cohorte 242 observationnelle portant sur 1942 femmes âgées de 75 à 85 ans, Fritel et al. 243 retrouvaient une altération de la posture, de l'équilibre et de la mobilité plus importante 244 dans le groupe de patientes présentant une incontinence urinaire [24]. Cette 245 constatation laissait penser qu'une prise en charge de rééducation plus globale 246 permettrait de prévenir et de corriger l'apparition d'une incontinence urinaire chez la 247 femme âgée. Morrisroe et al. dans une étude de cohorte longitudinale menée chez 328 248 femmes âgées de 60 à 93 ans retrouvait qu'une amélioration de la condition physique 249 était associé à une diminution de l'incidence annuelle d'une incontinence urinaire (OR $2500,69[0,5-0,95])$ [25]. D’autres auteurs ont étudié l'impact d'une rééducation posturale 251 utilisant la réalité virtuelle (Wii ${ }^{\circledR}$ Balance Board, StepMania, etc.) sur l'incontinence 252 urinaire, avec des résultats préliminaires encourageants [26]. En supposant une 253 participation cognitive à la survenue d'une incontinence urinaire, dans une étude 254 observationnelle portant sur 24 patientes souffrant d'IUM, Elliott et al. ont évalué 255 l'apport d'une thérapie cognitive (technique de rééducation virtuelle) en sus d'une 256 rééducation pelvi-périnéale conventionnelle. Ils retrouvaient une amélioration 257 significative des symptômes (questionnaires de qualité de vie), des performances 258 cognitives, de la musculature périnéale et de l'adhésion aux programmes de rééducation 259 [26]. A notre avis, cela montre que la rééducation est une prise en charge globale qui ne 260 doit pas être limitée au plancher pelvien. Cependant, ces nouvelles pistes de rééducation 261 incluant un travail sur la posture dans le traitement de l'incontinence urinaire n'ont pas 262 pu encore être évaluées sur un grand panel de patientes et nécessitent de futurs essais 263 contrôlés randomisés pour préciser leurs indications. 
Rééducation périnéale et incontinence urinaire du postpartum et de 3 à $38 \%$ à 3 mois du postpartum [1, 27-31]. Dans une mise à jour récente des

268 recommandations de l'ICI, Dumoulin et al. rappelaient l'utilité de la rééducation 269 pelvipérinéale dans le traitement d'une IUE dans le postpartum [16].

Concernant les patientes primipares présentant une IU à 3 mois du postpartum,

deux essais randomisés, retrouvaient une amélioration significative des symptômes d'incontinence à 6 mois et 9 mois mais pas d'effet à un an [32,33]. De même, dans une population toutes parités confondues, un récent essai randomisé avec un suivi à 12 ans, a été mené pour évaluer l'efficacité à long terme d'une rééducation pelvipérinéale supervisée par rapport à une prise en «charge conventionnelle » chez des patientes présentant une IU à 3 mois du postpartum. Ils retrouvaient un bénéfice de la rééducation à un an (60\% d'IU versus 69\%) sans que celui-ci ne persiste 12 ans plus tard [34]. Dumoulin et al, ont analysé les critères prédictifs de réussite de la rééducation 279 pelvipérinéale pendant 8 semaines avec une séance par semaine chez les femmes 280 présentant une IUE persistante à 3 mois du postpartum. Il s'agissait d'une analyse 281 secondaire d'un essai randomisé visant à comparer deux types de programme de 282 rééducation (abdominale et périnéale vs. périnéale). Ils retrouvaient qu'un faible tonus 283 des MPP (mesuré par dynamométrie) avant l'instauration d'une rééducation était 284 corrélé à un meilleur taux de succès [35].

285 Plusieurs essais randomisés s'étaient déjà focalisés sur l'intérêt d'une rééducation 286 pelvipérinéale en cours de grossesse pour la prévention de l'apparition d'une IUE au cours de la grossesse et dans le postpartum [32,35,36]. Morkved et al. retrouvaient une 288 incidence d'IUE plus faible à 36 semaines d'aménorrhée $(32 \%$ vs. $48 \%, p=0,007)$ et à 3 
mois du postpartum ( $20 \%$ vs. $32 \%, p=0,18)$ dans un groupe de femmes nullipares ayant bénéficiées d'une rééducation pelvipérinéale supervisée (12 semaines) par rapport à

291 celle n'en ayant pas eu [36]. Reilly et al. retrouvaient des résultats similaires chez des

292 patientes nullipares présentant une hypermobilité cervicale [36]. Plus récemment deux

293 essais randomisés ne retrouvaient pas d’effet bénéfique de la rééducation pelvipérinéale 294 préventive (basée sur le renforcement des MPP) sur la prévalence de l'incontinence du 295 postpartum. Hilde et al., dans un essai randomisé, comparaient l'effet d'une rééducation 296 pelviperinéale supervisée débutée a 6 semaines du postpartum et de simple conseil de 297 rééducation périnéale chez des patientes primipares présentant ou non une 298 incontinence urinaire. A 6 mois, il n'existait pas de différence significative en terme de 299 prévalence d'incontinence urinaire entre les deux groupes [38]. Fritel et al., dans un 300 essai randomisé, comparaient l'effet d’une rééducation pelvipérinéale supervisée 301 prénatale (débutée à 6 mois de grossesse) et de simples conseils écrits sur la survenue 302 d'une incontinence urinaire chez des patientes nullipares. A 12 mois du postpartum, les 303 auteurs ne montraient pas de différences significatives entre les deux groupes 304 concernant la prévalence de l'incontinence urinaire quel que soit le statut de continence 305 de la patiente lors de l'inclusion [39]. A notre avis, la rééducation est indiquée en cas de 306 symptômes urinaires significatifs en période prénatale ou postnatale, mais n'a pas 307 d'indication pour la prévention de l'incontinence urinaire [40].

Rééducation pelvipérinéale et prolapsus

Les études observationnelles montrent qu'une mauvaise fonction des MPP 311 (manque de force, manque de contraction automatique, manque de capacité à relâcher) 312 est associée à un risque plus important de prolapsus sans qu'il soit possible de préciser 313 le sens de l'association [41-43]. Selon la dernière méta-analyse de la Cochrane publiée 
314 en 2011, la rééducation pelvipérinéale par renforcement des MPP est efficace dans la

315 réduction des symptômes périnéaux associés au prolapsus [44]. Cette analyse, incluant

3164 essais contrôlés randomisés dont le niveau de risque de biais était important, ne

317 permettait pas d'apporter un niveau de preuve de haute qualité. Elle ne permettait

318 également pas de conclure sur l'efficacité à moyen terme et à long terme de la

319 rééducation. L'effet de correction anatomique (ou la réduction du grade du prolapsus)

320 apporté par la rééducation reste incertain. Dans l'essai de Braekken et al qui incluait 109

321 femmes, 19\% des femmes du groupe rééducation (renforcement des MPP) ont vu le 322 grade de leur prolapsus s'améliorer contre 4\% dans le groupe contrôle [45]. Hagen et al.

323 ont réalisé l'essai POPPY (randomisé multicentrique) visant à évaluer l'efficacité de la

324 rééducation dans le traitement du prolapsus à 12 mois. Le groupe traitement (225

325 patientes) bénéficiait d’une rééducation pelvipérinéale consistant en 5 séances

326 personnalisées en face à face avec un rééducateur réparties sur 16 semaines associées à

327 un programme de contraction périnéale quotidien, elles étaient encouragées à effectuer

3283 séances de 10 contractions longues et 50 contractions rapides par jour [46]. Le groupe

329 contrôle (222 patientes) recevait un dépliant d'information sur le prolapsus.

330 L'évaluation des MPP était basée sur le modèle PERFECT. Les caractéristiques des

331 patientes étaient similaires dans les deux groupes avec une majorité de patientes

332 présentant un prolapsus grade 2 (73\% dans le groupe rééducation vs. $78 \%$ dans le

333 groupe témoin). Les patientes étaient revues à 6 mois pour une évaluation clinique en

334 aveugle (POP-Q) et recevaient un questionnaire par voie postale de symptômes à 6 et 12

335 mois. Le critère de jugement principal était l'auto-évaluation par les patientes des

336 symptômes associés au prolapsus à 12 mois. Les auteurs rapportaient une amélioration

337 significativement plus importante des symptômes à 6 et 12 mois dans le groupe

338 traitement. A 12 mois, à la question «comment trouvez-vous votre prolapsus par 
rapport au début de l'étude ? », 57\% des patientes du groupe traitement contre $45 \%$ du groupe contrôle répondaient «mieux » $(\mathrm{p}=0,01)$. En revanche, dans les deux groupes, il

341 n'existait pas de différence significative entre la correction anatomique du prolapsus à

342 l'évaluation initiale et à 6 mois. L'essai de Wiegersma et al., montre une amélioration des

343 symptômes du prolapsus mesuré par le questionnaire PFDI-20 qui est plus importante

344 dans le groupe renforcement des MPP (score PFDI-20 moyen à 47 après versus 65

345 avant) que dans le groupe contrôle (51 après versus 59 avant). Cependant ce résultat (9

346 points de différence entre les 2 groupes) était inférieur à celui qui était considéré 347 comme cliniquement significatif (15 points de différence) par les auteurs [47]. A notre 348 avis, l'effet de la rééducation sur les symptômes du prolapsus génital reste donc modéré 349 à faible. La rééducation peut être utile dans cette indication quand les symptômes sont 350 modérés et qu'un autre traitement (pessaire ou chirurgie) n'est pas envisagé

351 Il faut souligner qu'il existe une grande diversité des programmes de 352 renforcement musculaire étudiés dans la littérature. La durée du programme variait de 3538 à 26 semaines [5,35,39,45]. La fréquence des séances encadrées par un rééducateur 354 variait entre 1 fois par semaine et 1 fois toutes les 6 semaines [16,33,35,37,39]. Le 355 nombre des séances variait de 3 à 20 [16,33]. La durée de chaque séance variait de 30 à 35670 minutes $[5,34,38]$. Les séances pouvaient être en groupe ou individuelles $357[5,16,35,39,45]$. De nombreux programme privilégiaient l'intensité de la contraction, 358 d'autres l'endurance, certains associaient au renforcement musculaire, les cônes, le 359 biofeedback, ou l'électrostimulation. Cependant l'encadrement par le rééducateur 360 comprenait dans tous les cas une visite d'inclusion au cours de laquelle un enseignement 361 d'anatomie et de conscientisation périnéale étaient prodigués et des visites de contrôle 362 permettant de corriger les défauts et surtout d'encourager les femmes à poursuivre leur 363 traitement. A chaque fois, il était demandé aux femmes de continuer des contractions à 
364 domicile, par exemple des exercices de 8 à 12 contractions à intensité maximale répétés

3653 fois par jour $[9,33,38]$.

$366 \quad$ Conclusion

367 La rééducation périnéale encadrée par un rééducateur est un traitement efficace, à court 368 terme, pour réduire les symptômes d'incontinence urinaire ou de prolapsus génital. Elle 369 peut être utilisée et est indiquée quand il existe une plainte clinique. Elle ne prévient pas 370 la survenue de symptômes chez un sujet asymptomatique. Elle est indiquée en première 371 intention dans l'incontinence urinaire d'effort. La durée de son effet n'est pas bien 372 connue. Elargir les techniques de rééducation pelvipérinéale en incluant une approche 373 cognitive, comportementale et posturale semble une piste intéressante. Des travaux 374 futurs sont utiles pour préciser les critères prédictifs de l'efficacité de la rééducation et 375 les meilleures modalités de rééducation en fonction du bilan initial.

376

\section{Conflits d'intérêt}

Les auteurs ne rapportent pas de conflits d'intérêt. 
[1] Fritel X, Ringa V, Quiboeuf E, Fauconnier A. Female urinary incontinence, from pregnancy to menopause: a review of epidemiological and pathophysiological findings. Acta Obstet Gynecol Scand 2012;91:901-10. doi:10.1111/j.1600-0412.2012.01419.x. [2] Hermieu J-F, Denys P, Fritel X. [Critical review of guidelines for female urinary incontinence diagnosis and treatment]. Prog Urol 2012;22:636-43. doi:10.1016/j.purol.2012.08.004.

390 [3] Fritel X, Fauconnier A, Bader G, Cosson M, Debodinance P, Deffieux X, et al. 391 Diagnosis and management of adult female stress urinary incontinence: guidelines for clinical practice from the French College of Gynaecologists and Obstetricians. Eur J Obstet Gynecol Reprod Biol 2010;151:14-9. doi:10.1016/j.ejogrb.2010.02.041.

[4] Deffieux X, Billecocq S, Demoulin G, Rivain A-L, Trichot C, Thubert T. Mécanismes d'action de la rééducation périnéale pour l'incontinence urinaire de la femme. Prog Urol 2013;23:491-501. doi:10.1016/j.purol.2013.04.002.

[5] Dumoulin C, Peng Q, Stodkilde-Jorgensen H, Shishido K, Constantinou C. Changes in levator ani anatomical configuration following physiotherapy in women with stress urinary incontinence. J Urol 2007;178:970-7; quiz 1129. doi:10.1016/j.juro.2007.05.023.

[6] Dumoulin C, Hay-Smith EJC, Mac Habée-Séguin G. Pelvic floor muscle training versus no treatment, or inactive control treatments, for urinary incontinence in women. Cochrane Database Syst Rev 2014;5:CD005654. doi:10.1002/14651858.CD005654.pub3.

[7] Dumoulin C, Hay-Smith J. Pelvic floor muscle training versus no treatment, or inactive control treatments, for urinary incontinence in women. Cochrane Database Syst Rev 2010:CD005654. doi:10.1002/14651858.CD005654.pub2.

[8] Dumoulin C, Hay-Smith J, Habée-Séguin GM, Mercier J. Pelvic floor muscle training versus no treatment, or inactive control treatments, for urinary incontinence in women: A short version Cochrane systematic review with meta-analysis. Neurourol Urodyn 2014. doi:10.1002/nau.22700.

[9] Labrie J, Berghmans BLCM, Fischer K, Milani AL, van der Wijk I, Smalbraak DJC, et al. Surgery versus Physiotherapy for Stress Urinary Incontinence. N Engl J Med 2013;369:1124-33.

[10] Herderschee R, Hay-Smith EJC, Herbison GP, Roovers JP, Heineman MJ. Feedback or biofeedback to augment pelvic floor muscle training for urinary incontinence in women. Cochrane Database Syst Rev 2011:CD009252. doi:10.1002/14651858.CD009252.

[11] Di Gangi Herms AM, Veit R, Reisenauer C, Herms A, Grodd W, Enck P, Stenzl A, Birbaumer N. Functional imaging of stress urinary incontinence. Neuroimage 2006;29:267-75. .

[12] Imamura M, Abrams P, Bain C, Buckley B, Cardozo L, Cody J, et al. Systematic review and economic modelling of the effectiveness and cost-effectiveness of nonsurgical treatments for women with stress urinary incontinence. Health Technol Assess 2010;14:1-188, iii - iv. doi:10.3310/hta14400.

[13] Herbison GP, Dean N. Weighted vaginal cones for urinary incontinence. Cochrane Database Syst Rev 2013;7:CD002114. doi:10.1002/14651858.CD002114.pub2.

[14] Moore K, Dumoulin C, Bradley C, et al. Adult Conservative management. In: Abrams P, Cardoza L, Khoury AE, Wein A, editors. International consultation on urinary incontinence. 5th edition. Plymbridge, UK: Health Publications Ltd.; 2013. pp. 1101-95 
[15] Lopès P, Rimbault F, Scheffler M, André C, Cappelletti MC, Marès P. Etude prospective multicentrique randomisée évaluant l'intérêt de l'électrostimulation intravaginale à domicile après rééducation périnéale pour incontinence urinaire. Gynecol Obstet Fertil 2014;42:779-86.

[16] Dumoulin C, Hunter KF, Moore K, Bradley CS, Burgio KL, Hagen S, et al. Conservative management for female urinary incontinence and pelvic organ prolapse review 2013: Summary of the 5th international consultation on incontinence. Neurourol Urodyn 2014. doi:10.1002/nau.22677. [17] Sherburn M, Bird M, Carey M, Bø K, Galea MP. Incontinence improves in older women after intensive pelvic floor muscle training: an assessor-blinded randomized controlled trial. Neurourol Urodyn 2011;30:317-24. doi:10.1002/nau.20968.

[18] Wallace SA, Roe B, Williams K, Palmer M. Bladder training for urinary incontinence in adults. Cochrane Database Syst Rev 2004:CD001308. doi:10.1002/14651858.CD001308.pub2.

[19] Smith MD, Coppieters MW, Hodges PW. Postural response of the pelvic floor and abdominal muscles in women with and without incontinence. Neurourol Urodyn

448 [20] Griffiths D, Derbyshire S, Stenger A, Resnick N. Brain control of normal and overactive bladder. J Urol 2005;174:1862-7.

[21] Griffiths D, Tadic SD, Schaefer W, Resnick NM. Cerebral control of the bladder in normal and urge-incontinent women. Neuroimage 2007;37:1-7.

[22] Bakker E, Fayt C. Spécificité de la prise en charge de l'urgenturie et de l'incontinence. Réalités en Gyn-Obs 2011;(155):1-4

[23] Baker J, Costa D, Guarino JM, Nygaard I. Comparison of mindfulness-based stress reduction versus yoga on urinary urge incontinence: a randomized pilot study. with 6month and 1-year follow-up visits. Female Pelvic Med Reconstr Surg 2014;20:141-6. doi:10.1097/SPV.0000000000000061.

[24] Fritel X, Lachal L, Cassou B, Fauconnier A, Dargent-Molina P. Mobility impairment is associated with urge but not stress urinary incontinence in community-dwelling older women: results from the Ossébo study. BJOG 2013;120:1566-72. doi:10.1111/14710528.12316.

[25] Morrisroe SN, Rodriguez LV, Wang P-C, Smith AL, Trejo L, Sarkisian CA. Correlates of 1-year incidence of urinary incontinence in older Latino adults enrolled in a community-based physical activity trial. J Am Geriatr Soc 2014;62:740-6. doi:10.1111/jgs.12729.

[26] Elliott V, de Bruin ED, Dumoulin C. Virtual reality rehabilitation as a treatment approach for older women with mixed urinary incontinence: a feasibility study. Neurourol Urodyn 2014. doi:10.1002/nau.22553.

[27] Wijma J, Weis Potters AE, Tinga DJ, Aarnoudse JG. The diagnostic strength of the 24-h pad test for self-reported symptoms of urinary incontinence in pregnancy and after childbirth. Int Urogynecol J Pelvic Floor Dysfunct 2008;19:525-30. doi:10.1007/s00192007-0472-z.

[28] Stanton SL, Kerr-Wilson R, Harris VG. The incidence of urological symptoms in normal pregnancy. Br J Obstet Gynaecol 1980;87:897-900.

[29] Eason E, Labrecque M, Marcoux S, Mondor M. Effects of carrying a pregnancy and of method of delivery on urinary incontinence: a prospective cohort study. BMC Pregnancy Childbirth 2004;4:4. doi:10.1186/1471-2393-4-4.

[30] Pauls RN, Occhino JA, Dryfhout V, Karram MM. Effects of pregnancy on pelvic floor dysfunction and body image; a prospective study. Int Urogynecol J Pelvic Floor 
481 [31] Viktrup L, Lose G, Rolff M, Barfoed K. The symptom of stress incontinence caused by pregnancy or delivery in primiparas. Obstet Gynecol 1992;79:945-9. [32] Sampselle CM, Miller JM, Mims BL, Delancey JO, Ashton-Miller JA, Antonakos CL. Effect of pelvic muscle exercise on transient incontinence during pregnancy and after birth. Obstet Gynecol 1998;91:406-12.

[33] Ahlund S, Nordgren B, Wilander E-L, Wiklund I, Fridén C. Is home-based pelvic floor muscle training effective in treatment of urinary incontinence after birth in primiparous women? A randomized controlled trial. Acta Obstet Gynecol Scand 2013;92:909-15. doi:10.1111/aogs.12173. [34] Glazener CMA, MacArthur C, Hagen S, Elders A, Lancashire R, Herbison GP, et al. Twelve-year follow-up of conservative management of postnatal urinary and faecal incontinence and prolapse outcomes: randomised controlled trial. BJOG 2014;121:11220. doi:10.1111/1471-0528.12473.

[35] Dumoulin C, Bourbonnais D, Morin M, Gravel D, Lemieux M-C. Predictors of success for physiotherapy treatment in women with persistent postpartum stress urinary incontinence. Arch Phys Med Rehabil 2010;91:1059-63. doi:10.1016/j.apmr.2010.03.006.

[36] Mørkved S, Bø K, Schei B, Salvesen KA. Pelvic floor muscle training during pregnancy to prevent urinary incontinence: a single-blind randomized controlled trial. Obstet Gynecol 2003;101:313-9.

501 [37] Reilly ETC, Freeman RM, Waterfield MR, Waterfield AE, Steggles P, Pedlar F. 502 Prevention of postpartum stress incontinence in primigravidae with increased bladder 503 neck mobility: a randomised controlled trial of antenatal pelvic floor exercises. BJOG 504 2002;109:68-76.

505 [38] Hilde G, Stær-Jensen J, Siafarikas F, Ellström Engh M, Bø K. Postpartum pelvic 506 floor muscle training and urinary incontinence: a randomized controlled trial. Obstet Gynecol 2013;122:1231-8. doi:10.1097/AOG.0000000000000012.

508 [39] Fritel X, Guilhot-Gaudeffroy J, de Tayrac R, Savary D, Deffieux X, Cotte L, 509 Fauconnier A. Prevention of postnatal urinary incontinence by antenatal pelvic floor 510 muscle exercises, secondary per protocol analysis of the 3PN (Prenatal Pelvic Floor 511 Prevention) randomized trial. Neurourol Urodyn 2013;32:528-9.

512 [40] Fritel X. Pour ou contre la rééducation périnéale du post-partum ? Gynecol Obstet 513 Fertil 2008;36:824-5.

514 [41] Braekken IH, Majida M, Ellström Engh M, Holme IM, Bø K. Pelvic floor function is 515 independently associated with pelvic organ prolapse. BJOG 2009;116:1706-14. 516 doi:10.1111/j.1471-0528.2009.02379.x.

517 [42] Slieker-ten Hove M, Pool-Goudzwaard A, Eijkemans M, Steegers-Theunissen R, 518 Burger C, Vierhout M. Pelvic floor muscle function in a general population of women 519 with and without pelvic organ prolapse. Int Urogynecol J 2010;21:311-9. 520 doi:10.1007/s00192-009-1037-0.

521 [43] Moen MD, Noone MB, Vassallo BJ, Elser DM, Urogynecology Network. Pelvic floor 522 muscle function in women presenting with pelvic floor disorders. Int Urogynecol J Pelvic 523 Floor Dysfunct 2009;20:843-6. doi:10.1007/s00192-009-0853-6.

524 [44] Hagen S, Stark D. Conservative prevention and management of pelvic organ 525 prolapse in women. Cochrane Database Syst Rev 2011:CD003882. 526 doi:10.1002/14651858.CD003882.pub4.

527 [45] Braekken IH, Majida M, Engh ME, Bø K. Can pelvic floor muscle training reverse 528 pelvic organ prolapse and reduce prolapse symptoms? An assessor-blinded, 
529 randomized, controlled trial. Am J Obstet Gynecol 2010;203:170.e1-7.

530 doi:10.1016/j.ajog.2010.02.037.

531 [46] Hagen S, Stark D, Glazener C, Dickson S, Barry S, Elders A, Frawley H, Galea MP, 532 Logan J, McDonald A, McPherson G, Moore KH, Norrie J, Walker A, Wilson D. 533 Individualised pelvic fl oor muscle training in women with pelvic organ prolapse 534 (POPPY): a multicentre randomised controlled trial. Lancet 2014;383:796-806.

535 [47] Wiegersma M, Panman CM, Kollen BJ, Berger MY, Lisman-Van Leeuwen Y, Dekker $536 \mathrm{JH}$. Effect of pelvic floor muscle training compared with watchful waiting in older 537 women with symptomatic mild pelvic organ prolapse: randomised controlled trial in 538 primary care. BMJ 2014;349:g7378. doi: 10.1136/bmj.g7378. 